\section{Seasonal competition between mould spores and respiratory viruses}

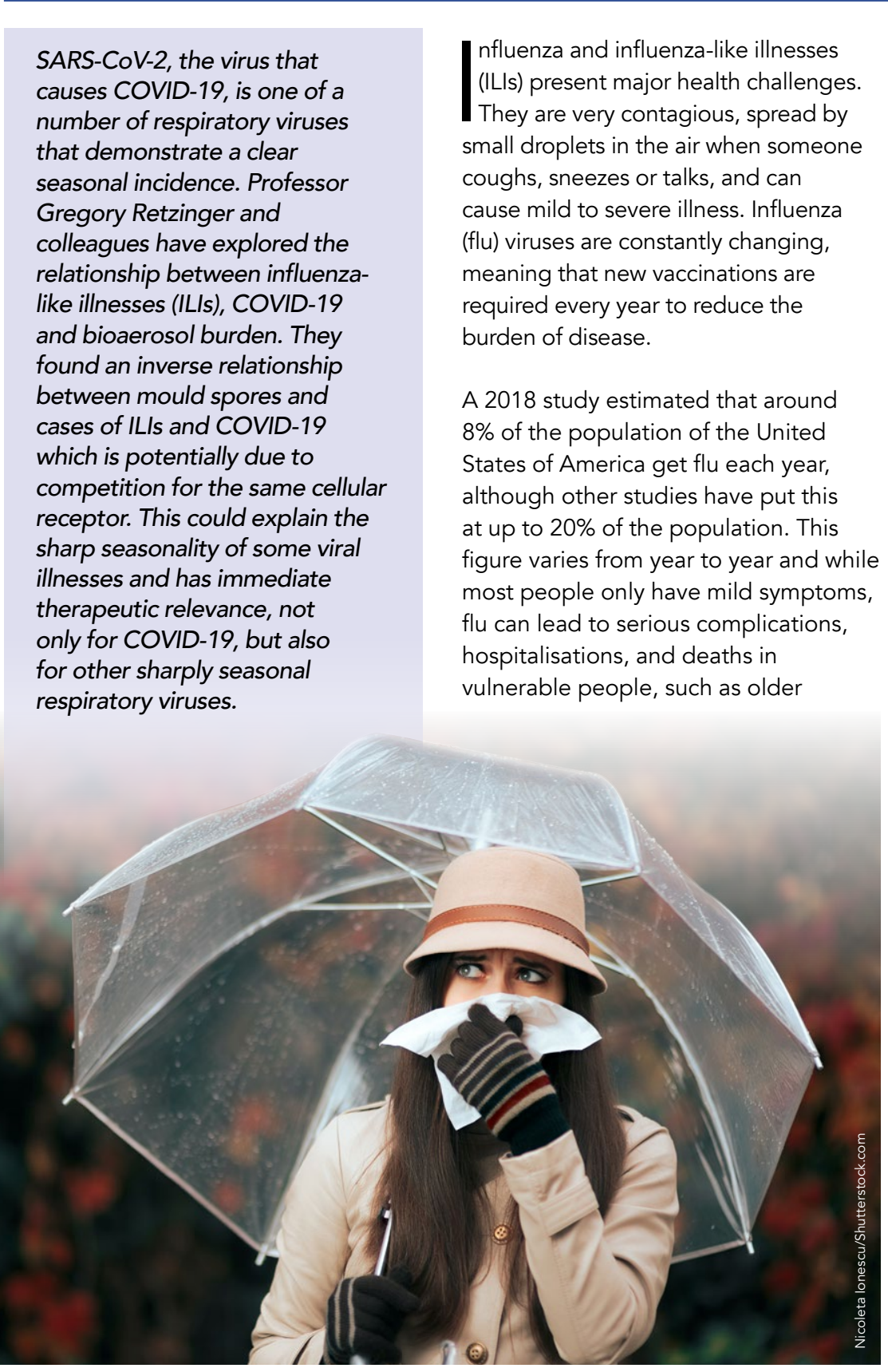
environment for their growth. people, young children and people with certain

As winter moves into spring, the challenges presented by seasonal flu associated with an increase in poll evels. Mould spores also increase during spring, summer and early autumn and this may be why people with allergies suffer worse symptoms during these times of the year. Moulds prefer damp conditions which is why spring weather provides an optima

SARS-COV-2, the virus that causes COVID-19, also appears to have a seasonal pattern, for reasons not The Netherlands have indicated an inverse relationship between the seasonal incidence of ILI and pollen count. Meanwhile, a decrease in ILls alongside rising pollen counts has been observed in emergency departments in Chicago, USA. In a new study, Gregory Retzinger, Professor of Pathology at Northwestern University Chicago, studied the seasonal incidence and bioaerosol burden.

POLLEN AND MOULD SPORES Whilst previous studies have only looked this study also induded mollen and ILIs, this study also included mould spores as these are known seasor

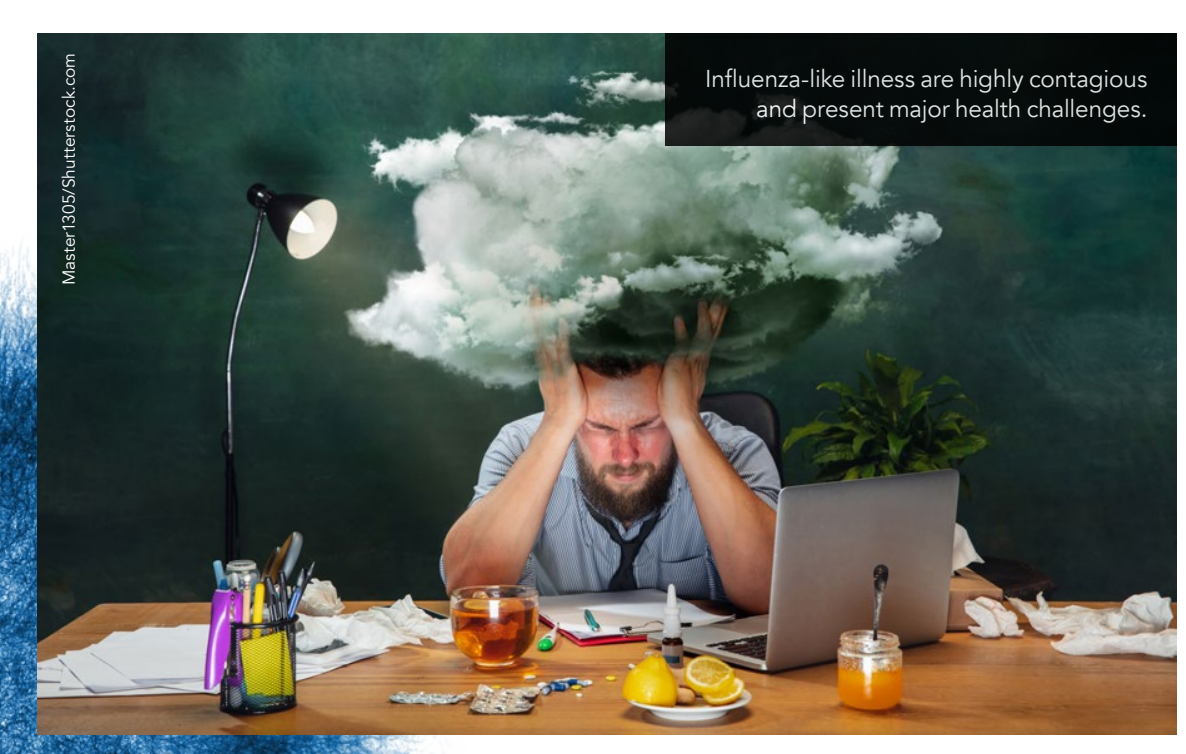

Evidence from the traps revealed that total bioaerosol count (pollen and September and fell sharply in following months The majoity of the following months. The majorty of the mould spores, with a peak seen during

PATTERNS IN ILIS COVID-19 CASES from the Chicago Department of Public to July 2020 for 23 hospitals that consistently reported cases of IIIs during the whole study period. COVID-19 data were collected from the Chicago Data. Portal, which provides information about COVID-19 cases, deaths and rates of esting across the local community.

Data analysis showed that cases of ILI present to emergency departments in Chicago in a 12-month cycle. The peak occurs at the start of the year in February and the lowest rate of ILls was reported in August, although they never completely disappear.

Analysing the COVID-19 data was slightly more challenging, as robust reporting several months after the pandemic 2020 However, the study suggests a decrease

When levels of mould spores are high, TLR4 may be binding to these instead of other targets, especially respiratory viruses.

late September and falling rapidly until in COVID-19 cases between May and September, followed by an increase. Whi during the year, with the first main smaller peak in late August.

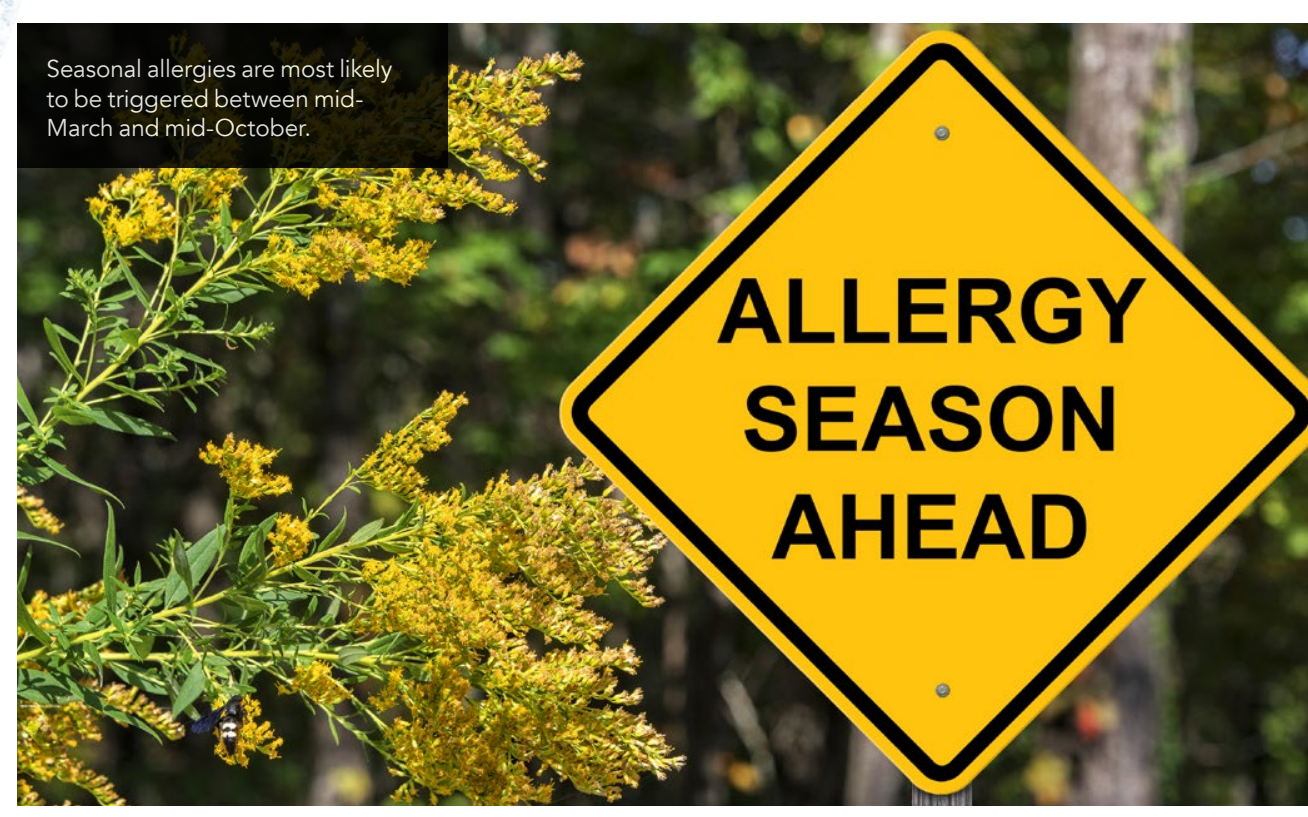


Cell Surface

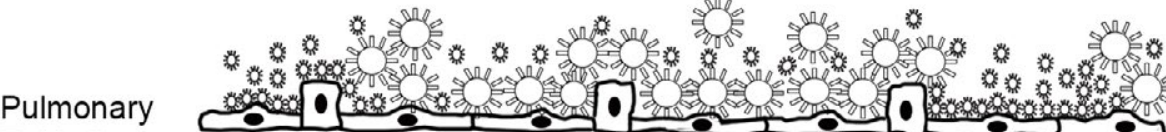
Epithelium

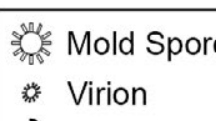

$$
\text { 密 }
$$

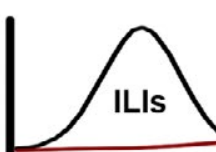

Winter

S

environment available to be transmitted - it is not clear how local restrictions and
lockdown rules impacted the pattern of lockdown rules impacted the pattern of
reported COVID-19 cases. There may reported COVID-19 cases. There may
also have been cases of COVID-19 that were not recorded, given the novel nature of the disease and the fact that many individuals remained asymptomatic.

\section{COMPETING FOR ATTENTION}

While the start of pollen season appears to do not seem to remain particularly high when II and COVID-19 cases are low. Th is in contrast to mould spores, which seem to increase continuously in a first-order pattern, i.e., they increase as COVID-19 cases decrease. As moulds have higher atmospheric concentration and a longer season than pollens, they are more like to play a role in the reduction of ILIs. This effect could be because bioaerosols stimulate the immune system in a way tha makes it more resilient to viral infection. However, Professor Retzinger focused on a more direct effect: that they are directly competing with viruses through
receptor in the immune system.

COVID-19 and ILI are caused by viruses that bind to receptors on cells in the body enabling them to enter the cell, replicate mould spores studied by the researchers were found to be echinulated, i.e., they have protuberances similar to those that enable flu and SARS-CoV-2 viruses to bind to cell receptors.

Previous studies in this area had focused on angiotensin-converting enzyme 2 (ACE-2) and its role in COVID-19, bu Retzinger considered Toll-like receptor 4 (TLR4) to be a better subject. TLR4 is a pattern recognition receptor that activates cells in the immune system. It from The Netherlands, showing that the

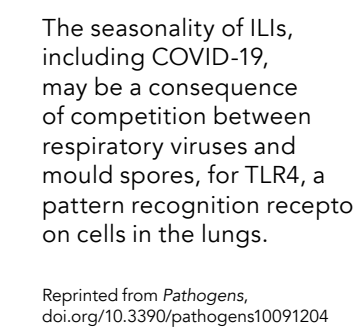

polysaccharide called lipopolysaccharide, a component of gram-negative bacteria.

It also plays a role in the inflammatory response triggered by sharply seasona respiratory viruses, and in our defence against multiple species of fungi. Cells
in the lungs (the main organ affected by in the lungs (the main organ affected by of TLR4 molecules on their surface. Furthermore, TLR4 binds the SARS-

CoV-2 spike protein with greater affinity than ACE-2.

Professor Retzinger proposes that, when levels of mould spores are high, TLR4 may be binding to these instead of other targets, meaning that respiratory viruses would be excluded - in effect the viruses from taking hold in our lungs

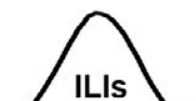

The results of this study suggest a complex interplay between respiratory viruses and bioaerosols that is dependent on seasonality.

Understanding this process could have a significant influence on our approaches to treating COVID-19a

CONCLUSIONS AND NEXT STEPS The data from Chicago confirm results incidence of ILls falls as pollen count rises. Further, Professor Retzinger and colleagues have shown that mould spores have the strongest inverse relationship with ILIs, i.e., levels of mould spores are highest throughout the spring and summer months

Professor Retzinger concludes that there is an inverse roltionship betwo the incidence of ILIs, including bioaerosols. He proposes that spores compete for a common receptor in the body, TLR4.

While an inverse relationship between pollen and expected given the time of year at which they occur, it is particularly interesting that a fall in mould spores is so strongly associated with higher levels of ILIs. It could be predicted that there would be more overlap betw mould spores and ILIs, due to the sonal closeness. COVID-19, and seasonal levels of
The results of this study suggest a complex interplay between respiratory viruses seasonality. Understanding more about this relationship will be critical for suggested treatments for COVID-19 and ILI.

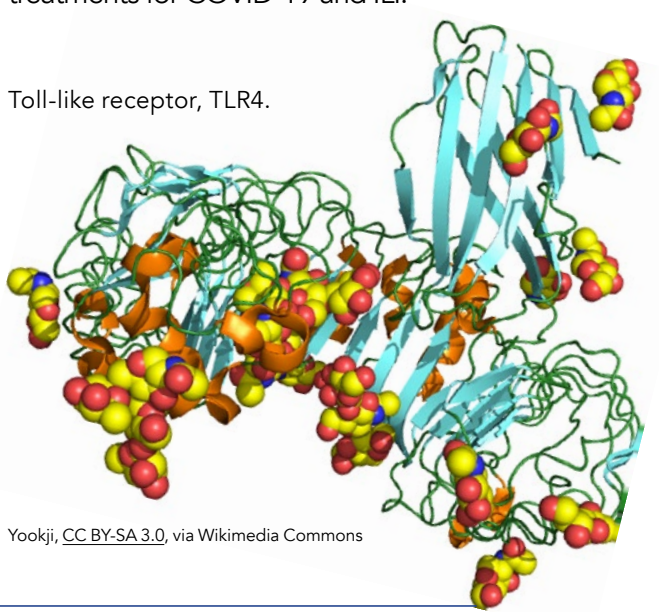

\section{Behind the Research} Professor Gregory Retzinger

\section{Research Objectives}

The influence of bioaerosols, including mould spores and pollens, on coronavirus infection.

\section{Detail}

Address

Department of Pathology, Feinberg School of Medicine, Dorthwestern University, Chicago, IL 60611, USA.

Bio

ollowing medical school, Gregory Retzinger completed a residency and a fellowship at the University of North Carolina at Chapel Hill, where he received a prestigious Lucille P Markey Biomedical Scholar Award. In 2011 he Medicine as Associate Chairman and Director of Clinical Pathology.

Funding

Department of Pathology Feinberg School of Medicine, Northwestern University.

\section{Collaborators}

Shah, Damien Retzinger, Andrew Retzinger, and Deborah Retzinger.

\section{References}

Shah, RB, Shah, RD, Retzinger, DG, Retzinger, AC, Retzinger DA \& Retzinger, GS, (2021). Competing Bioaerosols May Influence the Seasonality of Influenza-like Illnesses, including COVID-19. The Chicago Experience. Pathogens. 10(9), 1204. (Formerly, Confirmation of an Inverse Relationship betwe Bovid 19 . Ont and Influenza-like llinesses, Including (10.3390 of Mold Spores. medRxiv) Available at: doi.org/10.3390/pathogens10091204

\section{Personal Response}

What are your next steps to further understand the pathogenesis of COVID-19?

Given this new understanding, lam most interested in exploring the therapeutic efficacy of molecules that modulate or interfere with the activity and/or binding capacity of TLR4. Further, I feel quite strongly that the best

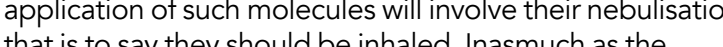
that is to say they should be inhaled. Inasmuch as the binding of viruses to TLR4 occurs at the environment-facing
side of the pulmonary tree, it stands to reason that maximal
disruption of that binding might also occur there.

MORFEINBERG NM Northwestern Northwestern University
Eeinberg School of Medicine

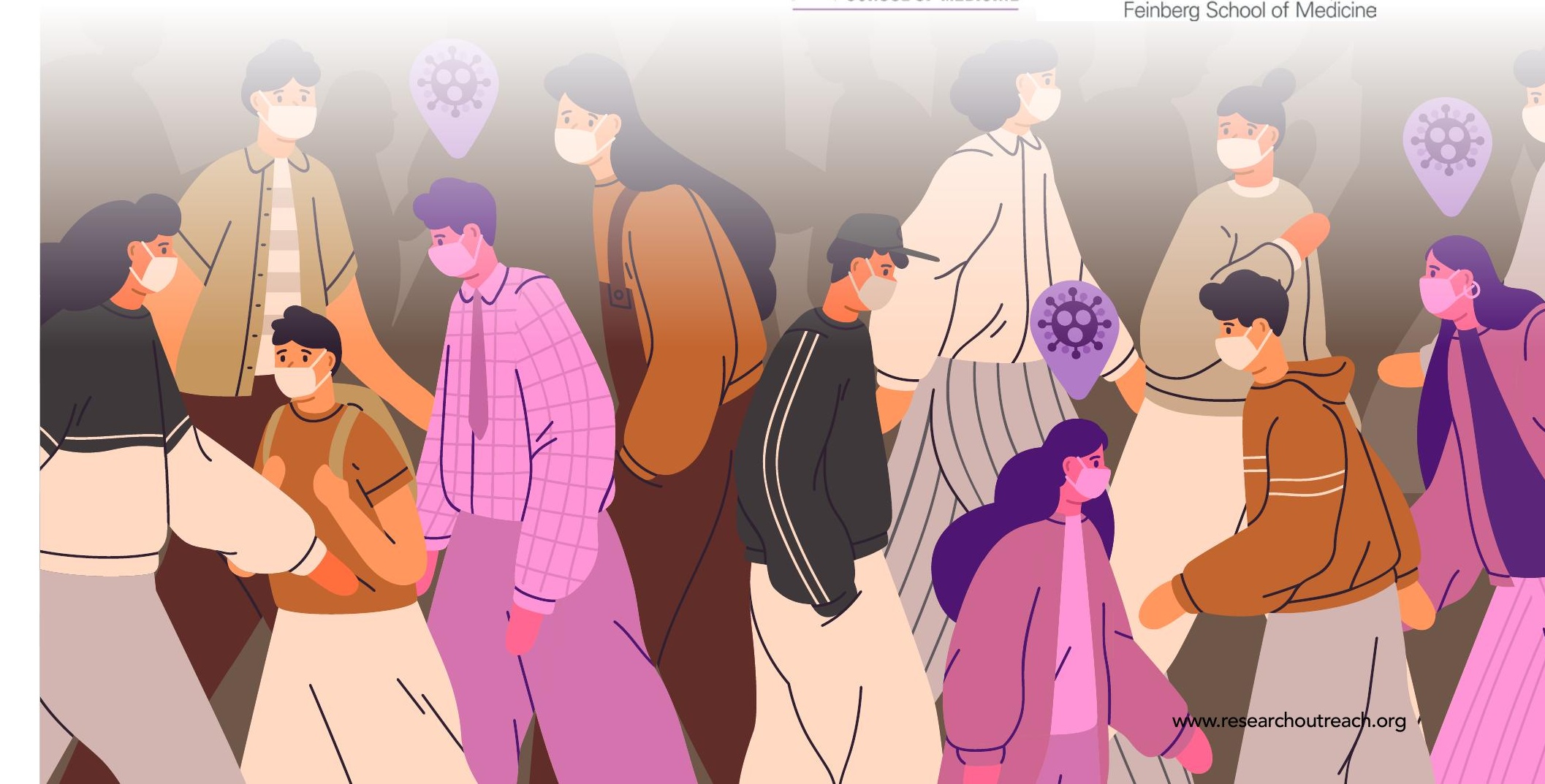

